\title{
Static Gauss-Bonnet black holes at large $D$
}

\author{
Bin Chen ${ }^{a, b, c}$ and Peng-Cheng $\mathbf{L i}^{a}$ \\ ${ }^{a}$ Department of Physics and State Key Laboratory of Nuclear Physics and Technology, \\ Peking University, \\ No.5 Yiheyuan Rd, Beijing 100871, P.R. China \\ ${ }^{b}$ Collaborative Innovation Center of Quantum Matter, \\ No. 5 Yiheyuan Rd, Beijing 100871, P.R. China \\ ${ }^{c}$ Center for High Energy Physics, Peking University, \\ No.5 Yiheyuan Rd, Beijing 100871, P.R. China \\ E-mail: bchen01@pku.edu.cn, wlpch@pku.edu.cn
}

ABStract: We study the static black holes in the large $D$ dimensions in the Gauss-Bonnet gravity with a cosmological constant, coupled to the Maxewell theory. After integrating the equation of motion with respect to the radial direction, we obtain the effective equations at large $D$ to describe the nonlinear dynamical deformations of the black holes. From the perturbation analysis on the effective equations, we get the analytic expressions of the frequencies for the quasinormal modes of charge and scalar-type perturbations. We show that for a positive Gauss-Bonnet term, the black hole could become unstable only if the cosmological constant is positive, otherwise the black hole is always stable. However, for a negative Gauss-Bonnet term, we find that the black hole could always be unstable. The instability of the black hole depends not only on the cosmological constant and the charge, but also significantly on the Gauss-Bonnet term. Moreover, at the onset of instability there is a non-trivial static zero-mode perturbation, which suggests the existence of a new nonspherically symmetric solution branch. We construct the non-spherical symmetric static solutions of the large $D$ effective equations explicitly.

KeYwords: Black Holes, Classical Theories of Gravity

ARXiv EPrint: 1703.06381 


\section{Contents}

1 Introduction 1

2 (Anti-)de Sitter charged Gauss-Bonnet black holes 3

$3 \quad$ Large $D$ effective equations $\quad 4$

4 Instability of de Sitter charged Gauss-Bonnet black hole 8

5 The case $\tilde{\alpha}<0 \quad 16$

$\begin{array}{llr}6 & \text { Summary } & 21\end{array}$

\section{Introduction}

Recently it has been found that black hole physics in Einstein's gravity can be efficiently investigated by using the $1 / D$ expansion in the near region of the black hole. The large $D$ expansion was first proposed by R. Emparan, R. Suzuki and K. Tanabe (EST) in [1] and subsequently developed in a series of papers [2-4]. The essence in the large $D$ expansion is that when the spacetime dimension is sufficiently large $D \rightarrow \infty$, the gravitational field of a black hole is strongly localized near its horizon due to the dominant radial gradient of the gravitational potential. As a result, for the decoupled quasinormal modes [5] the black hole can be effectively taken as a surface or membrane embedded in the background spacetime [6-11]. The membrane is described by the way it is embedded into the background spacetime, and its nonlinear dynamics is determined by the effective equations obtained by integrating the Einstein equations in the radial direction. Then the frequencies of the decoupled quasinormal modes of the black hole solutions can be obtained by performing the perturbation analysis of the effective equations. Furthermore, by solving the effective equations with different embedding of the membrane, one can construct different black hole solutions such as non-uniform black string [12] and black rings [11], and study numerically the final fate of their evolutions due to the instability $[10,13,14]$.

To understand the large $D$ expansion method better, it is valuable to extend the study to other gravity theories. One of most interesting generalization of Einstein gravity in higher dimensions is the Lovelock higher-curvature gravity of various orders. The most attractive feature of the Lovelock gravity is that its equations of motion are still the second order differential equations such that the fluctuations around the vacuum do not have ghost-like mode. Among all the Lovelock gravities, the second-order Lovelock gravity, the so called Einstein-Gauss-Bonnet gravity, is of particular interest. It includes the quadratic terms of the curvature tensors which appear as the leading-order correction in the low energy effective action of the heterotic string theory $[15,16]$. The exact spherically symmetric 
black hole solution of the Gauss-Bonnet gravity theory was discovered by Boulware and Deser [16] and independently by Wheeler [17](but without a cosmological constant). The construction of the Gauss-Bonnet black hole solution was later generalized to the case with an electric charge $[18,19]$.

The study of the Gauss-Bonnet black holes at large $D$ was initiated in [20]. The quasinormal modes of the black holes in the large $D$ limit have been computed carefully. It was found that the decoupled quasinormal modes, which characterize the information of the black hole, share the similar features as the ones in the Einstein gravity. The computation of the quasinormal modes in [20] relied on the master equations of the fluctuations. On the other hand, it turns out to be more efficient at large $D$ to study the black hole dynamics using the effective theory. In this paper, we would like to discuss the large $D$ effective theory of the Gauss-Bonnet black holes and study their instabilities.

The stability of the black hole under the perturbation is an important issue. It has been investigated for the Gauss-Bonnet black holes since their findings. For the asymptotically flat Gauss-Bonnet black holes it was found in [21, 22] that such black holes are unstable against gravitational perturbations in five and six dimensions but become stable in higher dimensions [23]. For a Gauss-Bonnet black hole with a positive cosmological constant, it was shown in [24] that the black holes becomes unstable in $D \geq 5$ dimensions at sufficiently large values of the cosmological constant. ${ }^{1}$ In [20], the instability of the asymptotically flat Gauss-Bonnet black holes at large $D$ has been discussed in two interesting limits. One limit is that the Gauss-Bonnet term can be treated as a small correction to the Einstein gravity, and the other one is to let the Gauss-Bonnet term be dominant. In both cases the GaussBonnet black holes are found to be stable. One unsolved question is the instability of the black hole for an arbitrary Gauss-Bonnet term. Moreover, the studies on the stability of the black hole have been focused on the case with a positive Gauss-Bonnet term, it would be interesting to consider the case with a negative Gauss-Bonnet term.

In the presence of the cosmological constant and the charge, the stability of the black hole at large $D$ can be very different. In [25], it was shown that the de Sitter ReissnerNordstrom black hole in the Einstein gravity becomes unstable against scalar-type gravitational perturbation when the charge is sufficient large. Moreover, it was found that there is a non-trivial zero-mode static perturbation at the threshold of the instability. The existence of such zero-mode indicates that there must be a non-spherical symmetric solution branch of static charged de Sitter black holes, whose specific form can be constructed by solving the large $D$ effective equations. In this work, we would like to investigate the effect of the cosmological constant and/or the charge on the stability of the Gauss-Bonnet black holes.

The remaining part of the paper is organized as follows. In section 2 we briefly review the (Anti-)de Sitter charged Gauss-Bonnet black holes. In section 3 we derive the large $D$ effective equations for the black holes, and in section 4 we perform the stability analysis of various black holes in the theory with a positive Gauss-Bonnet term. In section 5 we extend the stability analysis of the black holes to the case with a negative Gauss-Bonnet term. We end with some conclusion and discussions in section 6 .

\footnotetext{
${ }^{1}$ For instability of AdS-Gauss-Bonnet black holes see also [39, 40].
} 


\section{2 (Anti-)de Sitter charged Gauss-Bonnet black holes}

Let us consider the Gauss-Bonnet gravity with a positive cosmological constant, coupled to the Maxwell theory. Its action is given by

$$
S=\frac{1}{16 \pi G} \int d^{D} x \sqrt{-g}\left(R+\alpha\left(R_{\mu \nu \lambda \delta} R^{\mu \nu \lambda \delta}-4 R_{\mu \nu} R^{\mu \nu}+R^{2}\right)-\frac{(D-1)(D-2)}{L^{2}}-\frac{1}{4} F_{\mu \nu} F^{\mu \nu}\right) .
$$

Here $\alpha$ is the the Gauss-Bonnet coefficient. It is positive definite and inversely proportional to the string tension in the heterotic string theory [16]. However, we do not restrict ourselves to the case $\alpha \geq 0$ and allow it to be free parameter in this paper. The cosmological constant can be negative, which gives the black hole solution in AdS, but the form of the solution is similar.

From the action, we obtain the equations of motion for the metric

$$
\begin{aligned}
R_{\mu \nu}-\frac{1}{2} g_{\mu \nu} R= & -\frac{(D-1)(D-2)}{2 L^{2}} g_{\mu \nu}+\alpha\left(\frac{1}{2} g_{\mu \nu}\left(R_{\sigma \gamma \lambda \delta} R^{\sigma \gamma \lambda \delta}-4 R_{\lambda \delta} R^{\lambda \delta}+R^{2}\right)\right. \\
& \left.-2 R R_{\mu \nu}+4 R_{\mu \gamma} R_{\nu}^{\gamma}-4 R^{\gamma \delta} R_{\gamma \mu \nu \delta}-2 R_{\mu \gamma \delta \lambda} R_{\nu}^{\gamma \delta \lambda}\right) \\
& +\frac{1}{2}\left(F_{\mu \rho} F_{\nu}{ }^{\rho}-\frac{1}{4} g_{\mu \nu} F_{\rho \sigma} F^{\rho \sigma}\right),
\end{aligned}
$$

and the Maxwell equations

$$
\nabla^{\mu} F_{\mu \nu}=0
$$

where $F_{\mu \nu}=\partial_{\mu} A_{\nu}-\partial_{\nu} A_{\mu}$. The spherically symmetric charged de Sitter Gauss-Bonnet black hole has the metric [19]

$$
d s^{2}=-f(r) d t^{2}+f^{-1}(r) d r^{2}+r^{2} d \Omega_{D-2}^{2},
$$

where

$$
f(r)=1+\frac{r^{2}}{2 \tilde{\alpha}}\left(1-\sqrt{1+\frac{64 \pi G \tilde{\alpha} M}{(D-2) \Omega_{D-2} r^{D-1}}-\frac{2 \tilde{\alpha} Q_{e}^{2}}{(D-2)(D-3) r^{2 D-4}}+\frac{4 \tilde{\alpha}}{L^{2}}}\right),
$$

and the Maxwell field ${ }^{2}$

$$
A_{\mu} d x^{\mu}=\frac{Q_{e}}{(D-3) r^{D-3}} d t
$$

with

$$
\tilde{\alpha}=\alpha(D-3)(D-4) .
$$

In the following discussion we will assume that the largest root of $f(r)=0$ which corresponds to the outer horizon always exists. In the metric function $f(r), M$ is the mass of the black hole which can be expressed as

$$
M=\frac{(D-2) \Omega_{D-2} r_{+}^{D-3}}{16 \pi G}\left(1+\frac{\tilde{\alpha}}{r_{+}^{2}}\right) .
$$

\footnotetext{
${ }^{2}$ Note that here we use the convention in [26]. It seems that there is a typo for the field strength $F_{\mu \nu}$ in eq. (1.4b) in [19]. The correct form should be $F=\frac{Q}{4 \pi r^{D-2}} d t \wedge d r$.
} 
It is easy to see that when $1 / L \rightarrow 0$ and $Q_{e} \rightarrow 0, r_{+}$is just the horizon radius of the usual Gauss-Bonnet black hole.

In order to discuss the large $D$ expansion more conveniently, we introduce

$$
n=D-3,
$$

and

$$
\mathrm{R}=\left(\frac{r}{r_{0}}\right)^{n}
$$

where $r_{0}$ is a constant parameter and can be set to be unity. In order to see the effect of the gauge field on the solution in the large $D$ limit, we should replace $Q_{e}$ with another $\mathcal{O}(1)$ variable

$$
\widetilde{Q}=\frac{Q_{e}}{r_{+}^{n} \sqrt{2 n(n+1)}},
$$

in terms of which the gauge field can be written as

$$
A_{\mu} d x^{\mu}=\sqrt{\frac{2(n+1)}{n}} \widetilde{Q}\left(\frac{r_{+}}{r}\right)^{n} d t .
$$

In terms of $\mathrm{R}$, at the leading order of $1 / n$ expansion, $f(r)$ becomes

$$
f(\mathrm{R})=1+\frac{r_{0}^{2}}{2 \tilde{\alpha}}\left(1-\sqrt{\left.1+\frac{4 \tilde{\alpha}}{\mathrm{R}} \frac{r_{+}^{n}\left(1+\frac{\tilde{\alpha}}{r_{+}^{2}}\right)}{r_{0}^{n+2}}-\frac{4 \tilde{\alpha}}{\mathrm{R}^{2}} \frac{\widetilde{Q}^{2} r_{+}^{2 n}}{r_{0}^{2 n+2}}+\frac{4 \tilde{\alpha}}{L^{2}}\right)}\right.
$$

From the above expression we can see that if we use $Q_{e}$ instead of $\widetilde{Q}$, at the leading order of $1 / n$ expansion the gauge field term disappears. This fact suggests that the variable $\widetilde{Q}$ is more appropriate in the large $D$ expansion.

\section{$3 \quad$ Large $D$ effective equations}

In this section, we derive the large $D$ effective equations for the theory (2.1) and hence study the stabilities of the solutions. First of all, for the spherically symmetric metric solution (2.4) we can make the metric ansatz in terms of the ingoing Eddington-Finkelstein coordinates as

$$
d s^{2}=-A d v^{2}+2\left(u_{v} d v+u_{z} d z\right) d r-2 C_{z} d v d z+r^{2} G d z^{2}+r^{2} H^{2} d \Omega_{n}^{2},
$$

where $z$ is the inhomogeneous coordinate and can be interpreted as one of the coordinates of $d \Omega_{n+1}^{2}$. The generalization to the case with several inhomogeneous coordinates would be straightforward. It might also be possible to use this metric to describe Gauss-Bonnet black strings with appropriate embedding of the membrane, where $z$ is the direction the string extends along. In this article we will not pursue this interesting problem further but leave it for future work, instead we just focus on the black hole solutions.

The gauge filed ansatz is

$$
A_{\mu} d x^{\mu}=A_{v} d v+A_{z} d z
$$


As explained in [25], the $A_{r} d r$ term is omitted due to the fact that it does not contribute to effective equations at the leading order in the $1 / n$ expansion. For example in $F_{t r}=$ $\partial_{t} A_{r}-\partial_{r} A_{t}$, the first term is of $\mathcal{O}(1 / n)$ if we assume that $A_{r}=\mathcal{O}(1 / n)$, but the second term is of $\mathcal{O}(n)$ since $\partial_{r}=\mathcal{O}(n)$. The functions in the metric and gauge fields generally depend on $(v, \mathrm{R}, z)$ except that $G$ and $H$ depend only on $z$ because at the asymptotic infinity $\partial_{v}$ is a Killing vector. In order to do the $1 / n$ expansion properly we need to specify the large $D$ behaviors of these functions. Their large $n$ scalings are respectively

$$
A, u_{v}, A_{v}=\mathcal{O}(1), \quad u_{z}, C_{z}, A_{z}=\mathcal{O}(1 / n), \quad G=1+\mathcal{O}(1 / n)
$$

At the leading order, since $\partial_{r}=\mathcal{O}(n), \partial_{v}=\mathcal{O}(1)$ and $\partial_{z}=\mathcal{O}(1)$ the equations of motion only contain R-derivative so they can be solved by performing $\mathrm{R}$ integrations. The leading order solutions can be written as $^{3}$

$$
\begin{aligned}
A & =A_{0}^{2}+\frac{u_{v}^{2}}{L^{2}}+\frac{u_{v}^{2}}{2 \tilde{\alpha}}\left(1-\sqrt{\left.1+\frac{4 \tilde{\alpha}}{L^{2}}+\frac{4 \tilde{\alpha} m}{\mathrm{R} u_{v}^{2}}-\frac{4 \tilde{\alpha} q^{2}}{\mathrm{R}^{2} u_{v}^{2}}\right)}\right. \\
A_{v} & =\frac{\sqrt{2} q}{\mathrm{R}}, \quad A_{z}=-\frac{1}{n} \frac{\sqrt{2} p_{z} q}{m \mathrm{R}}, \quad u_{v}=\frac{A_{0}}{\sqrt{\frac{1-H^{\prime}(z)^{2}}{H(z)^{2}}-\frac{1}{L^{2}}}}, \quad G=1+\mathcal{O}\left(n^{-2}\right), \\
C_{z} & =\frac{1}{n} \frac{p_{z} u_{v}^{2}}{m}\left[-\left(\frac{1}{2 \tilde{\alpha}}+\frac{1}{L^{2}}\right)+\frac{1}{2 \tilde{\alpha}} \sqrt{1+\frac{4 \tilde{\alpha}}{L^{2}}+\frac{4 \tilde{\alpha} m}{\mathrm{R} u_{v}^{2}}-\frac{4 \tilde{\alpha} q^{2}}{\mathrm{R}^{2} u_{v}^{2}}}\right] .
\end{aligned}
$$

The integration functions in the solutions are the functions of $(v, z)$. In fact it is easy to see that in the limit $\tilde{\alpha} \rightarrow 0$ the above expressions reduce to the ones found in [25]. Moreover the effect of the Gauss-Bonnet term only appears in the functions $A$ and $C_{z}$, without appearing in other functions. In particular $C_{z}$ has a simple relation with $A$ as

$$
C_{z}=\frac{1}{n} \frac{p_{z}}{m}\left(-A+A_{0}^{2}\right)
$$

which has also been found in the Einstein gravity.

Comparing with the exact solution (2.13) we can read the physical meanings of the integration functions appearing in the leading order solutions: $m(v, z)$ and $q(v, z)$ are the mass density and the charge density respectively, $p_{z}(v, z)$ is a momentum density along $z$ direction, $A_{0}$ is a constant which is independent of $v$ and $z$, and $u_{z}$ is a shift vector on a $r=$ const. hypersurface. At the leading order $u_{z}$ can be gauged away so it vanishes identically. From the leading order form of the metric it is easy to find the outer and inner horizon radii of the dynamical black hole, which correspond to the two roots of $A=0$,

$$
\mathrm{R}_{ \pm}=\frac{m \pm \sqrt{m^{2}-4\left(A_{0}^{2}+\tilde{\alpha}\left(\frac{A_{0}^{2}}{u_{v}}+\frac{u_{v}}{L^{2}}\right)^{2}\right) q^{2}}}{2\left(A_{0}^{2}+\tilde{\alpha}\left(\frac{A_{0}^{2}}{u_{v}}+\frac{u_{v}}{L^{2}}\right)^{2}\right)}
$$

\footnotetext{
${ }^{3}$ Note that the $\mathcal{O}(1 / n)$ term in $G$ vanishes identically at the leading order in the $1 / n$ expansion.
} 
At the next-to-leading order, we could obtain the equations for $m(v, z), q(v, z)$ and $p_{z}(v, z)$. They can be taken as the effective equations for the charged de Sitter GaussBonnet black holes. These equations are

$$
\begin{gathered}
\partial_{v} q-\frac{u_{v} H^{\prime}(z)}{H(z)} \partial_{z} q+\frac{A_{0}^{2} H^{\prime}(z)}{H(z)} \frac{p_{z} q}{m}=0, \\
\partial_{v} m-\frac{u_{v} H^{\prime}(z)}{H(z)} \partial_{z} m+\frac{A_{0}^{2} H^{\prime}(z)}{H(z)} p_{z}=0, \\
\partial_{v} p_{z}-\frac{u_{v} H^{\prime}(z)}{m H(z)} \frac{2 A_{0}^{2} u_{v}^{2}\left(L^{2}+4 \tilde{\alpha}\right) \mathrm{R}_{+}-\left(2 \tilde{\alpha} u_{v}^{2}+L^{2}\left(u_{v}^{2}-2 A_{0}^{2} \tilde{\alpha}\right)\right) m}{2 u_{v}^{2} \tilde{\alpha}+L^{2}\left(u_{v}^{2}+2 A_{0}^{2} \tilde{\alpha}\right)} \partial_{z} p_{z} \\
+\left[1+\frac{2 u_{v}^{3}\left(\left(A_{0}^{2} L^{2}+4 A_{0}^{2} \tilde{\alpha}\right) \mathrm{R}_{+}-\left(L^{2}+2 \tilde{\alpha}\right) m\right) p_{z}}{L^{2} u_{v}^{2}+2 A_{0}^{2} L^{2} \tilde{\alpha}+2 u_{v}^{2} \tilde{\alpha}} \frac{H^{\prime}(z)}{H(z) m^{2}}\right] \partial_{z} m \\
-\left[\frac{2 u_{v}^{4} \tilde{\alpha}+L^{2}\left(u_{v}^{4}-2 A_{0}^{2} u_{v}^{2} \tilde{\alpha}\right)+4 A_{0}^{2}\left(A_{0}^{2} L^{2}+u_{v}^{2}\right) \tilde{\alpha} H(z)^{2}}{u_{v}\left(2 u_{v}^{2} \tilde{\alpha}+L^{2}\left(u_{v}^{2}+2 A_{0}^{2} \tilde{\alpha}\right)\right) H(z)^{2}}\right. \\
\left.+\frac{2 A_{0}^{2}\left(L^{2}+4 \tilde{\alpha}\right)\left(-L^{2} u_{v}^{3}+\left(A_{0}^{2} L^{2} u_{v}+u_{v}^{3}\right) H(z)^{2}\right) \mathrm{R}_{+}}{L^{2}\left(2 u_{v}^{2} \tilde{\alpha}+L^{2}\left(u_{v}^{2}+2 A_{0}^{2} \tilde{\alpha}\right)\right) H(z)^{2} m}-\frac{A_{0}^{2} H^{\prime}(z)}{H(z)} \frac{p_{z}}{m}\right] p_{z}=0 .
\end{gathered}
$$

Besides, in order to describe the embedding of the membrane in the background spacetime, we need to do " $(D-1)+1$ " decomposition on a $r=$ const. surface. Then the momentum constraint of the decomposition at the leading order gives

$$
\frac{d}{d z} \frac{A_{0}}{\sqrt{\frac{1-H^{\prime}(z)^{2}}{H(z)^{2}}-\frac{1}{L^{2}}}}=0 .
$$

The constraint suggests that $u_{v}$ can be regarded as a constant.

By assuming $m(v, z)=m(z), q(v, z)=q(z)$ and $p_{z}(v, z)=p_{z}(z)$ it is straightforward to find a static solution from these effective equations. From (3.9) and (3.10), we obtain

$$
p_{z}(z)=\frac{u_{v}}{A_{0}^{2}} m^{\prime}(z), \quad q(z)=K m(z),
$$

where $K$ is a constant. Plugging (3.13) into (3.11) and setting $m(z)=e^{P(z)}$, we have the equation for $P(z)$

$$
\begin{aligned}
& P^{\prime \prime}(z) \\
& +\left[\frac{A_{0}^{2} H(z)}{L^{2} u_{v}^{2} H^{\prime}(z)} \frac{2 u_{v}^{2}\left(A_{0}^{2} L^{2}+u_{v}^{2}\right)\left(L^{2}+\tilde{\alpha}\right) S_{+}+2 L^{2}\left(A_{0}^{2} L^{2}+u_{v}^{2}\right) \tilde{\alpha}}{2 A_{0}^{2} u_{v}^{2}\left(L^{2}+4 \tilde{\alpha}\right) S_{+}-L^{2} u_{v}^{2}+2\left(A_{0}^{2} L^{2}-u_{v}^{2}\right) \tilde{\alpha}}-\frac{1}{H(z) H^{\prime}(z)}\right] P^{\prime}(z)=0,
\end{aligned}
$$

where

$$
S_{+}=\frac{1+\sqrt{1-4\left(A_{0}^{2}+\tilde{\alpha}\left(\frac{A_{0}^{2}}{u_{v}}+\frac{u_{v}}{L^{2}}\right)^{2}\right) K^{2}}}{2\left(A_{0}^{2}+\tilde{\alpha}\left(\frac{A_{0}^{2}}{u_{v}}+\frac{u_{v}}{L^{2}}\right)^{2}\right)} .
$$

The functions $H(z)$ and $A_{0}$ are given through an embedding of the leading order solution into a background spacetime, and they should satisfy the condition (3.12). As long as we know $H(z)$ and $A_{0}$, we can determine the function $m(z)$. 
There are two special cases worth noticing. The first one is the limit $\tilde{\alpha}=0$. In this case (3.14) is used to describe the de Sitter charged black holes. At the extremal limit $\mathrm{R}_{+}=\mathrm{R}_{-}$, (3.14) is not valid any more. This fact can be seen directly from the denominator of the first term in the square bracket in (3.14)

$$
L^{4} u_{v}^{4} H^{\prime}(z) \sqrt{1-4 A_{0}^{2} K^{2}}
$$

which becomes zero in the extremal limit. In other words, the extremal point is singular for the de Sitter Reissner-Nordstrom black holes, similar to the case for the charged black ring [27]. In contrast, for the Gauss-Bonnet gravity, this singular behavior does not arise, because (3.14) is always regular for the physical solution. The other special case is the limit $A_{0}=0$. In this case it is obvious (3.13) is not valid, neither is (3.14). From the effective equations, in this limit we obtain $m(z)=$ const., $q(z)=$ const. and $p_{z}(z)=0$. Hence in this special case, no inhomogeneous solution exists.

In this static case $\mathrm{R}_{+}$is proportional to $m(z)$ and $\partial_{v}$ becomes the Killing vector. Consequently the surface gravity $\kappa$ can be expressed in terms of $S_{+}$in (3.15)

$$
\begin{aligned}
\kappa & =\left.\frac{n}{2} \frac{\mathrm{R} \partial_{\mathrm{R}} A}{u_{v}}\right|_{\mathrm{R}_{+}} \\
& =C \frac{n}{2 u_{v}} \frac{1}{1+2 \tilde{\alpha}\left(\frac{A_{0}^{2}}{u_{v}^{2}}+\frac{1}{L^{2}}\right)},
\end{aligned}
$$

with

$$
C=\frac{S_{+}-2 K^{2}}{S_{+}^{2}} .
$$

As $C$ is a constant, so is the surface gravity.

It was interestingly noted in $[6,7,13]$ that the stationary large $D$ black holes are the solutions of an elastic theory. For example, for the neutral static black holes the effective membrane embedded in the background must satisfy the equation

$$
\sqrt{-g_{v v}\left(1-v^{2}\right)} \mathcal{K}=2 \kappa
$$

where $\mathcal{K}$ is the trace of the extrinsic curvature of the membrane, $v$ represents the Lorentz boost on the membrane, $\kappa$ is just the surface gravity of the black hole and $g_{v v}$ is the redshift factor on the membrane. Especially for the static black holes in the Minkowski background $\sqrt{-g_{v v}}=1$, the membrane amounts to a spherical soap bubble. For Gauss-Bonnet black holes, the static solutions we obtained above are also the solutions of an elastic theory, but the equation is not as simple as the one (3.19). For example, if we treat $\tilde{\alpha}$ as a small quantity and keep its leading order, for the neutral case $K=0$, we have

$$
\sqrt{-g_{v v}} \mathcal{K}=\frac{n A_{0}^{2}}{u_{v}}+\frac{n u_{v} \tilde{\alpha}}{L^{4}}
$$

However, from (3.17) after setting $K=0$ we find

$$
2 \kappa=\frac{n A_{0}^{2}}{u_{v}}+\left(\frac{n u_{v}}{L^{4}}-\frac{A_{0}^{4}}{u_{v}^{3}}\right) \tilde{\alpha} .
$$


Obviously, these two equations cannot be equal generally unless $A_{0}=0$, which would lead to a trivial solution. Nevertheless, we still can write the static solutions in a elastic form, that is

$$
\sqrt{-g_{v v}} \mathcal{K}=\text { constant }
$$

\section{Instability of de Sitter charged Gauss-Bonnet black hole}

By embedding $H(z)$ and $A_{0}$ into the de Sitter spacetime, the de Sitter charged GaussBonnet black hole is obtained as a static solution of the effective equations. The embedding in the spherical coordinates is given by

$$
H(z)=\sin z, \quad A_{0}=\sqrt{1-\frac{1}{L^{2}}} .
$$

In order to keep $A_{0}$ non-negative we demand $L \geq 1$. Then the de Sitter charged GB black hole is given by a static solution

$$
p_{z}(v, z)=0, \quad q(v, z)=Q, \quad m(v, z)=1-\frac{1}{L^{2}}+\tilde{\alpha}+Q^{2} .
$$

Here we just set the horizon radius to be unity $R_{+}=1$ for convenience, so (4.2) is related to $(2.13)$ by

$$
Q^{2}=\tilde{Q}^{2} r_{+}^{2 n}, \quad 1-\frac{1}{L^{2}}+\tilde{\alpha}+Q^{2}=r_{+}^{n}\left(1+\frac{\tilde{\alpha}}{r_{+}^{2}}\right),
$$

and $K$ in (3.13) is related to $Q$ by

$$
K=\frac{Q}{1-\frac{1}{L^{2}}+\tilde{\alpha}+Q^{2}} .
$$

Besides, as the radius $R_{+}$must be positive, the physical solution requires that

$$
Q^{2} \leq 1-\frac{1}{L^{2}}+\tilde{\alpha}
$$

where the extremal case $\mathrm{R}_{+}=\mathrm{R}_{-}$corresponds to the saturated case $Q^{2}=1-\frac{1}{L^{2}}+\tilde{\alpha}$.

Now consider the perturbations around the static solution (4.2) with the ansatz

$$
m(v, z)=\left(1-\frac{1}{L^{2}}+\tilde{\alpha}+Q^{2}\right)\left(1+\epsilon e^{-i \omega v} F_{m}(z)\right), \quad q(v, z)=Q\left(1+\epsilon e^{-i \omega v} F_{q}(z)\right),
$$

and

$$
p_{z}(v, z)=\epsilon e^{-i \omega v} F_{z}(z)
$$

In order to make the above expressions simpler, let us introduce

$$
\bar{m}(v, z)=\frac{m(v, z)}{A_{0}^{2}}, \quad \bar{q}(v, z)=\frac{q(v, z)}{A_{0}}, \quad \bar{Q}=\frac{Q}{A_{0}}, \quad \bar{\alpha}=\frac{\tilde{\alpha}}{A_{0}^{2}},
$$

then the condition (4.5) becomes

$$
\bar{Q}^{2} \leq 1+\bar{\alpha}
$$


and eq. (4.6) becomes

$$
\bar{m}(v, z)=\left(1+\bar{\alpha}+\bar{Q}^{2}\right)\left(1+\epsilon e^{-i \omega v} F_{m}(z), \quad \bar{q}(v, z)=\bar{Q}\left(1+\epsilon e^{-i \omega v} F_{q}(z)\right) .\right.
$$

We have two kinds of the perturbations, the charge perturbation and the gravitational perturbation. The charge perturbation is defined by $F_{m}(z) \neq F_{q}(z)$, which describes the fluctuation with a net charge. The gravitational perturbations which describe density fluctuation are uniquely decomposed into scalar, vector and tensor type. They can be expanded in terms of the harmonic functions of different types. For the most interesting scalar-type perturbation $F_{m}(z)=F_{q}(z)$, which is related to the spherical harmonics $\mathbb{Y}_{\ell}$ on $S^{n+1}$. At a large $n, z=\pi / 2$ we know $^{4} \mathbb{Y}_{\ell} \sim \cos ^{\ell} z$.

To read the quasinormal modes of the perturbations, we need to impose appropriate boundary conditions on the perturbations. The perturbations should satisfy the outgoing boundary condition at the asymptotical infinity and the ingoing boundary condition at the horizon. The latter condition has naturally been achieved as we have taken the ingoing Eddington-Finkelstein coordinates. The former condition requires that at large $\mathrm{R}$

$$
\delta g_{\mu \nu}=\mathcal{O}\left(\mathrm{R}^{-1}\right)
$$

which obviously holds here. So plugging (4.7) and (4.10) into the effective equations (3.9), (3.10) and (3.11), we obtain the quasinormal mode for the charge perturbation

$$
\omega_{c}=-i \ell
$$

so the charge perturbation is stable. This result is the same as the case without a GaussBonnet term [9, 25] For the scalar-type gravitational perturbation we obtain the quasinormal mode condition as

$$
\begin{aligned}
& \frac{2\left(\ell+L^{2}\left(\omega^{2}-\ell+i \omega \ell\right)\right)\left(1+\bar{Q}^{2}+\bar{\alpha}\right)}{L^{2}}+\frac{1}{L^{4}+2 L^{2}\left(L^{2}-1\right) \bar{\alpha}}\left(8 i(\omega+i \ell)(-1+\ell) \bar{\alpha}\left(-1+\bar{Q}^{2}+\bar{\alpha}\right)\right. \\
& +2 i L^{4}(\omega+i \ell)\left(-2-4 \bar{\alpha}\left(1+\bar{Q}^{2}+\bar{\alpha}\right)+\ell\left(1+\bar{\alpha}+2 \bar{\alpha}^{2}+\bar{Q}(-1+2 \bar{Q})\right)\right) \\
& \left.+4 L^{2}(-i \omega+\ell) \bar{\alpha}\left(-\ell+\bar{Q}^{2}(-4+3 \ell)-4 \bar{\alpha}+3 \ell \bar{\alpha}\right)\right)=0 .
\end{aligned}
$$

This is a quadratic equation in $\omega$, which can be solved analytically. One important feature of the above equation is that the linear term in $\omega$ is purely imaginary, so the solution is of the form

$$
\omega_{ \pm}=-i \omega_{i} \pm \omega_{r}
$$

where $\omega_{i}$ is a positive real number, and $\omega_{r}$ is the square root of something $\omega_{r}=\sqrt{W}$. More precisely, the solution is

$$
\begin{aligned}
\omega_{ \pm}= & \frac{1}{L^{2}\left(1+\bar{Q}^{2}+\bar{\alpha}\right)\left(L^{2}+2 \bar{\alpha}\left(L^{2}-1\right)\right)}[ \\
& \left.-i(\ell-1)\left(L^{4}+2\left(L^{2}-1\right)\left(1-\bar{Q}^{2}+L^{2}\left(1+\bar{Q}^{2}\right)\right) \bar{\alpha}+2\left(L^{2}-1\right)^{2} \bar{\alpha}^{2}\right) \pm \sqrt{W(L, \bar{Q}, \bar{\alpha})}\right]
\end{aligned}
$$

\footnotetext{
${ }^{4}$ This can be seen from the definition of the spherical harmonics $\Delta_{S^{n+1}} \mathbb{Y}_{\ell}=-\ell(\ell+n) \mathbb{Y}_{\ell}$ where $\Delta_{S^{n+1}}=$ $\sin ^{2-n} z \frac{\partial}{\partial z} \sin ^{n-2} z \frac{\partial}{\partial z}+\sin ^{-2} z \Delta_{S^{n}}$. At $z=\pi / 2$ and in the large $n$ limit, it is easy to get that $\mathbb{Y}_{\ell} \sim \cos ^{\ell} z$.
} 
where

$$
\begin{aligned}
W(L, \bar{Q}, \bar{\alpha})= & -(\ell-1)^{2}\left(L^{4}+2\left(L^{2}-1\right)\left(1-\bar{Q}^{2}+L^{2}\left(1+\bar{Q}^{2}\right)\right) \bar{\alpha}+2\left(L^{2}-1\right)^{2} \bar{\alpha}^{2}\right)^{2} \\
& +L^{2} \ell\left(1+\bar{Q}^{2}+\bar{\alpha}\right)\left(L^{2}+2\left(L^{2}-1\right) \bar{\alpha}\right)\left(2\left(3+\bar{Q}^{2}(2 \ell-1)+2 \ell(\bar{\alpha}-1)-\bar{\alpha}\right) \bar{\alpha}\right. \\
& -L^{2}\left(1+\bar{Q}^{2}(1-4 \bar{\alpha}+6 \ell \bar{\alpha})+\bar{\alpha}(5-4 \bar{\alpha}+\ell(6 \bar{\alpha}-2) \bar{\alpha})\right) \\
& \left.+L^{4}(\ell-1)\left(1+\bar{\alpha}+2 \bar{\alpha}^{2}+\bar{Q}^{2}(2 \bar{\alpha}-1)\right)\right) .
\end{aligned}
$$

In the limit $\bar{\alpha} \rightarrow 0$ the solutions reproduce the ones for the de Sitter Reissner-Nordstrom black hole in [25]. When $W<0$, the frequency of the quasinormal mode becomes purely imaginary, and especially $\omega_{+}=i\left(\sqrt{-W}-\omega_{i}\right)$ may have a positive imaginary part, suggesting the black hole is unstable. The necessary condition that $\omega_{+}$has a positive imaginary part is

$$
\begin{aligned}
& \left.2\left(3+\bar{Q}^{2}(2 \ell-1)\right)+2 \ell(\bar{\alpha}-1)-\bar{\alpha}\right) \bar{\alpha}+L^{4}(\ell-1)\left(1+\bar{\alpha}+2 \bar{\alpha}^{2}+\bar{Q}^{2}(2 \bar{\alpha}-1)\right) \\
& -L^{2}\left(1+\bar{Q}^{2}(1-4 \bar{\alpha}+6 \ell \bar{\alpha})+\bar{\alpha}(5-4 \bar{\alpha}+\ell(6 \bar{\alpha}-2) \bar{\alpha})<0 .\right.
\end{aligned}
$$

Asymptotically flat Gauss-Bonnet black hole. Firstly, let us consider the asymptotically flat case which corresponds to $L \rightarrow \infty$. For the neutral case $\bar{Q}=0$, we easily obtain the frequencies of the scalar-type quasinormal mode as

$$
\omega_{ \pm}=\frac{-i(\ell-1)\left(1+2 \bar{\alpha}+2 \bar{\alpha}^{2}\right) \pm \sqrt{(\ell-1)\left[(1+2 \bar{\alpha}(1+\bar{\alpha}))^{2}-\ell \bar{\alpha}^{2}\right]}}{(1+\bar{\alpha})(1+2 \bar{\alpha})} .
$$

In the small and large $\bar{\alpha}$ limits, we get

$$
\omega_{ \pm}= \pm \sqrt{\ell-1}+i(\ell-1)
$$

which agree exactly with the ones found in [20], suggesting the black hole is stable. For a generic Gauss-Bonnet parameter, it has not been discussed in [20], as the analysis based on the master equations becomes rather difficult. Here it is fairly easy to read the quasinormal modes. In this case the necessary condition (4.17) becomes

$$
(\ell-1)\left(1+\bar{\alpha}+2 \bar{\alpha}^{2}\right)<0,
$$

which can never be satisfied. It turns out that there is no instability for the neutral GaussBonnet black hole at large $D$ for any value of the Gauss-Bonnet coefficient. The result is consistent with the one found in [23] that when the spacetime dimension is large enough, the Gauss-Bonnet black hole is always dynamically stable.

When the charge is taken into account, from (4.15) we find that

$\omega_{ \pm}=\frac{-i(\ell-1)\left(1+2\left(1+\bar{Q}^{2}\right) \bar{\alpha}+2 \bar{\alpha}^{2}\right) \pm \sqrt{(\ell-1)\left[\left(1+2\left(1+\bar{Q}^{2}\right) \bar{\alpha}+2 \bar{\alpha}^{2}\right)^{2}-\ell\left(\bar{Q}^{2}+\bar{\alpha}\right)^{2}\right]}}{(1+2 \bar{\alpha})\left(1+\bar{\alpha}+\bar{Q}^{2}\right)}$. 
The necessary condition (4.17) now becomes

$$
(\ell-1)\left(1+\bar{\alpha}+2 \bar{\alpha}^{2}+\bar{Q}^{2}(2 \bar{\alpha}-1)\right)<0
$$

since the charge is restricted to $\bar{Q}^{2} \leq 1+\bar{\alpha}$, the above condition can never be satisfied. Hence the imaginary parts of the frequencies are always negative. This suggests that the asymptotically flat charged Gauss-Bonnet black hole at large $D$ is always stable under the scalar-type gravitational perturbation, similar to the case of Reissner-Nordstrom black hole [9], although until now there is no numerical study to verify this. In addition, it would be interesting to note that at extremity, that is $\bar{Q}^{2}=1+\bar{\alpha}$, from (4.21) we find that $\omega_{+}$ becomes

$$
\omega_{+}=\frac{-i(\ell-1)(1+2 \bar{\alpha})+\sqrt{(\ell-1)\left[(1+2 \bar{\alpha})^{2}-\ell\right]}}{2(1+\bar{\alpha})} .
$$

For the extremal Reissner-Nordstrom black hole $\bar{\alpha}=0$ such that $\omega_{+}=0$. In contrast, for the extreaml Gauss-Bonnet black hole $\omega_{+} \neq 0$.

de Sitter Gauss-Bonnet black hole. When there is a positive cosmological constant, the stability of the Gauss-Bonnet black hole is different. In [24] the authors found that the Gauss-Bonnet black hole in de Sitter spacetime becomes unstable for $D \geq 5$ at sufficiently large values of the cosmological constant $\Lambda$, by using numerical analysis. This new kind of instability is called "the $\Lambda$-instability", because it does not take place for asymptotically flat spacetime. Here we can show this point explicitly in the large $D$ limit.

Consider first the neutral Gauss-Bonnet black hole without charge $\bar{Q}=0$, from (4.15) we obtain

$$
\begin{gathered}
\omega_{ \pm}=\frac{1}{L^{2}(1+\bar{\alpha})\left(L^{2}+\left(-1+L^{2}\right) \bar{\alpha}\right)}\left[-i(\ell-1)\left(L^{4}+2\left(-1+L^{4}\right) \bar{\alpha}+2\left(-1+L^{2}\right)^{2} \bar{\alpha}^{2}\right)\right. \\
\pm \sqrt{W(L, \bar{\alpha})}]
\end{gathered}
$$

where

$$
\begin{aligned}
W(L, \bar{\alpha})= & -(\ell-1)^{2}\left(L^{4}+2\left(-1+L^{4}\right) \bar{\alpha}+2\left(-1+L^{2}\right)^{2} \bar{\alpha}^{2}\right)^{2} \\
& +L^{2} \ell(1+\bar{\alpha})\left(L^{2}+2\left(-1+L^{2}\right) \bar{\alpha}\right)(2(3+2 \ell(-1+\bar{\alpha})-\bar{\alpha}) \bar{\alpha} \\
& \left.+L^{4}(-1+\ell)\left(1+\bar{\alpha}+2 \bar{\alpha}^{2}\right)+L^{2}(-1+\bar{\alpha}(-5+\ell(2-6 \bar{\alpha})+4 \bar{\alpha}))\right) .
\end{aligned}
$$

From this formula it is not hard to determine when the quasinormal mode frequency has a positive imaginary part so that the black hole becomes unstable. For example, when $L$ is sufficiently small, the term $W(L, \bar{\alpha})$ becomes negative, so the frequency $\omega_{+}$may have a positive imaginary part. Actually, the necessary condition (4.17) that the frequency $\omega_{+}$ has a positive imaginary part is

$$
2(3+2 \ell(-1+\bar{\alpha})-\bar{\alpha}) \bar{\alpha}+L^{4}(-1+\ell)\left(1+\bar{\alpha}+2 \bar{\alpha}^{2}\right)+L^{2}(-1+\bar{\alpha}(-5+\ell(2-6 \bar{\alpha})+4 \bar{\alpha}))<0 .
$$

From figure 1 we see that the quasinormal mode frequencies $\omega_{ \pm}$of de Sitter GaussBonnet black hole become purely imaginary when $1 / L>0.52$, and $\operatorname{Im}\left[\omega_{+}\right]>0$ when 

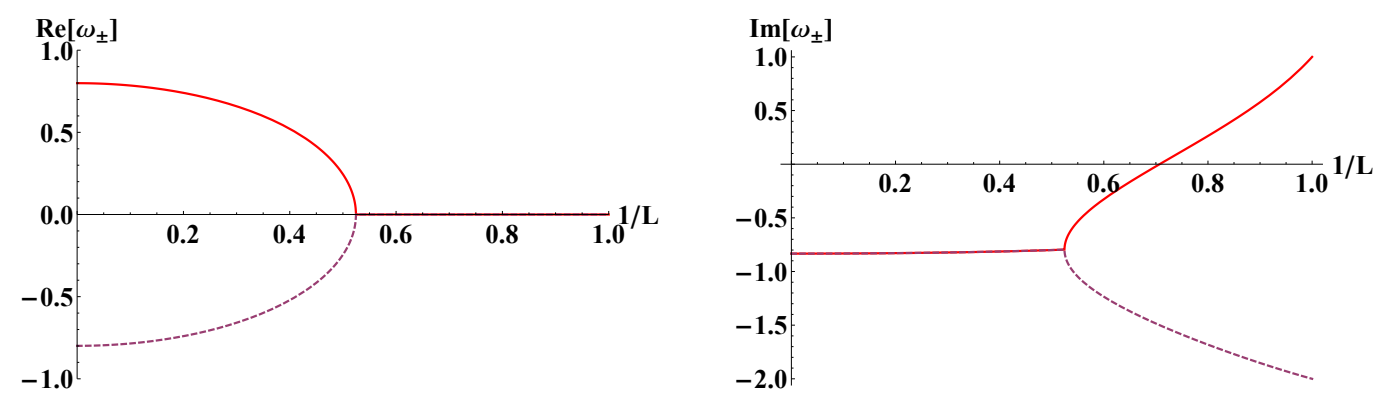

Figure 1. The quasinormal mode frequencies $\omega_{+}$(solid line) and $\omega_{-}$(dashed line) of the gravitational perturbation of de Sitter Gauss-Bonnet black hole with $\ell=2$ and $\bar{\alpha}=1$. The real part and the imaginary part are depicted in the left and right panel respectively. When $1 / L>1 / \sqrt{2} \approx 0.71$, the perturbation becomes unstable.
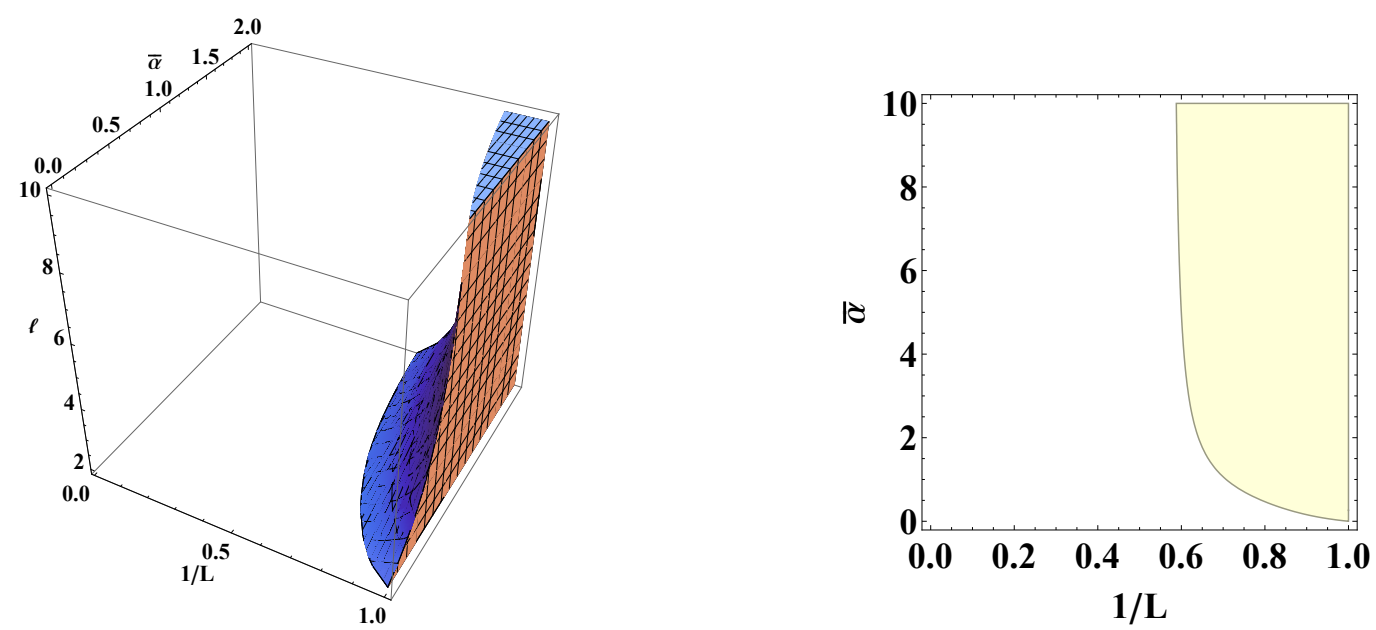

Figure 2. The unstable regions of the de Sitter Gauss-Bonnet black hole. The left panel shows the unstable region in $(L, \bar{\alpha}, \ell)$ space. Note that $\ell=2,3, \ldots$, here for simplicity we take $\ell$ to be continuous in this plot and in figure 3. The right panel shows the unstable region with $\ell=2$ in light yellow.

$1 / L>1 / \sqrt{2}$ suggesting the black hole could be unstable under a perturbation. In figure 2 we show the unstable region of the de Sitter Gauss-Bonnet black hole. It is easy to see that for a given $\bar{\alpha}$, when $L$ is smaller than a critical value $L_{\ell}$ the de Sitter Gauss-Bonnet black hole can always be unstable. This is consistent with the conclusion in [24] that when the cosmological constant is large enough, the black hole is unstable. From (4.24), in the limit $\bar{\alpha} \rightarrow \infty$, it is easy to obtain

$$
L_{\ell}=\sqrt{\frac{2 \ell-1}{\ell-1}}
$$

It would be interesting to compare the " $\Lambda$-instability" in the de Sitter Gauss-Bonnet black hole with the one in the de Sitter Reissner-Nordstrom black hole. In the latter case, for a fixed charge $\bar{Q}$, the instability appears when the cosmological constant obeys the 

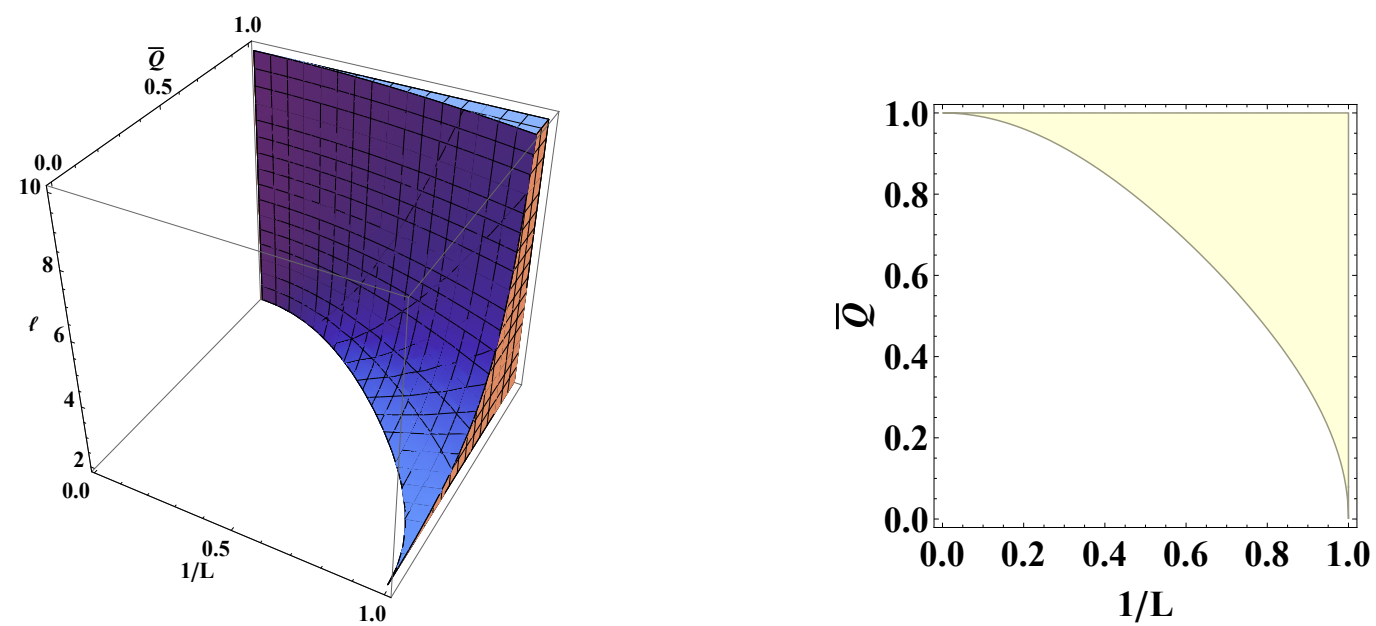

Figure 3. The unstable regions of the de Sitter Reissner-Nordstrom black hole. The left panel shows the unstable region in $(L, \bar{Q}, \ell)$ space. The right panel shows the unstable region with $\ell=2$ in light yellow.

following relation

$$
\frac{1}{L^{2}}>\frac{1}{L_{c}^{2}}=\frac{1-\bar{Q}^{2}}{1+\bar{Q}^{2}}(\ell-1)
$$

Obviously the presence of the charge can lower the critical value of the cosmological constant. On the other hand, for a fixed cosmological constant, when the charge is larger than the critical value [25]

$$
\bar{Q}_{\ell}=\sqrt{\frac{(\ell-1)-1 / L^{2}}{(\ell-1)+1 / L^{2}}},
$$

the de Sitter Reissner-Nordstrom black hole becomes unstable. In figure 3 we show the unstable region in the de Sitter Reissner-Nordstrom black hole. From figure 2 and figure 3 we can see that in both cases larger $\ell$ 's reduce the size of the unstable region, so $\ell=2$ corresponds to the most unstable mode.

Note that from the left panel of figure 2, we can see that the unstable region of the de Sitter Gauss-Bonnet black hole seems to extend to $L=1$ and $\bar{\alpha}=0$. However, $L=1$ leads to $A_{0}=0$, the form (4.8) is not correct any more. Instead we should use the original quantities (4.6) with $Q=0$ and $\tilde{\alpha}=0$. This obviously means that our solution breaks down in this limit since $m(v, z)=0$. This occurs also for the de Sitter Reissner-Nordstrom black hole [25] as observed in the left panel of figure 3. For the de Sitter Reissner-Nordstrom black hole $L=1$ is the Nariai limit but here the Nariai limit happens at $L=1 / \sqrt{1+\tilde{\alpha}}$ which is beyond the range we can touch as we require $L \geq 1$.

When both the charge and the Gauss-Bonnet term are taken into account, the instability of the black hole becomes complex. For simplicity, we take $\ell=2$ to illustrate their effects on the instability. In figure 4 we depict the unstable region of the de Sitter charged Gauss-Bonnet black hole in $(\bar{\alpha}, \bar{Q}, L)$ space. In order to show the effect of the charge, we compare the unstable regions with and without the charge in the left panel of figure 5 . We 


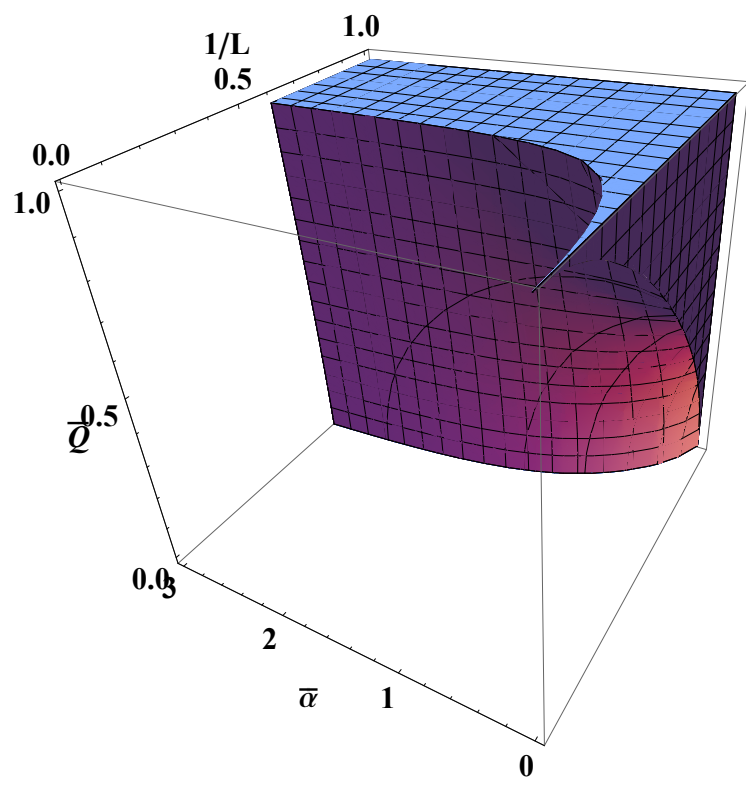

Figure 4. The unstable region of de Sitter charged Gauss-Bonnet black hole with $\ell=2$ is shown in $(\bar{\alpha}, \bar{Q}, L)$ space.
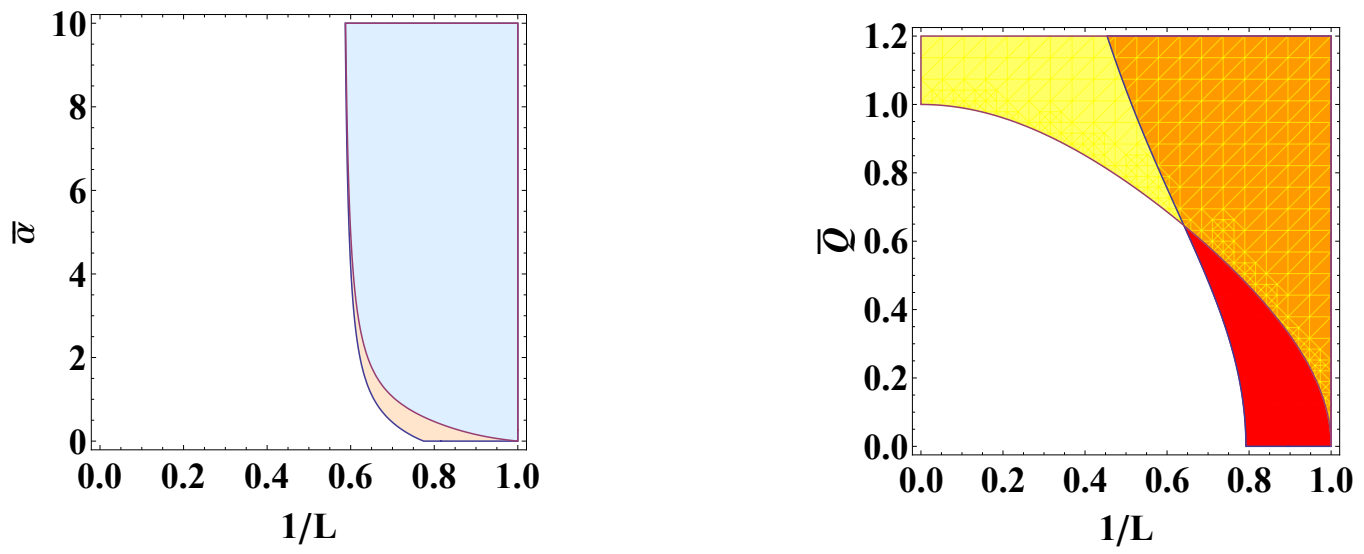

Figure 5. The unstable region of the de Sitter charged Gauss-Bonnet black hole with $\ell=2$. The left panel shows the unstable region in $(L, \bar{\alpha})$ plane, where the light blue region corresponds to $\bar{Q}=0$ and the light blue region plus the light orange region correspond to $\bar{Q}=0.5$. The right panel shows the unstable region in $(L, \bar{Q})$ plane, where the yellow region plus the orange region correspond to $\bar{\alpha}=0$ and the red region plus the orange region correspond to $\bar{\alpha}=0.5$.

can see that the presence of the charge enlarges the unstable region and lowers the critical value $L_{\ell}$. To show the effect of the Gauss-Bonnet term, we compare the unstable regions with and without the term in the right panel of figure 5. The presence of the Gauss-Bonnet coefficient $\bar{\alpha}$ enlarges the range of $\bar{Q}$ due to the relation $\bar{Q}^{2} \leq 1+\bar{\alpha}$, but the effect of $\bar{\alpha}$ on the instability of the black hole depends on the value of the cosmological constant. When $L$ is large, $\bar{\alpha}$ helps to stabilize the black hole, such that in some range of $L$ the black hole is always stable no matter what value the charge is taken. However, when $L$ is small, $\bar{\alpha}$ helps 

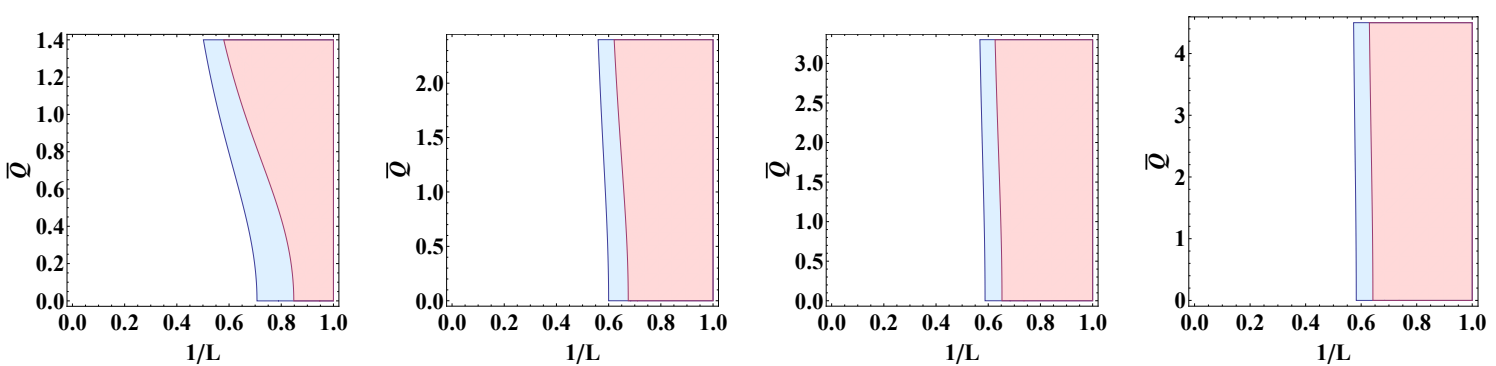

Figure 6. The unstable region of the de Sitter charged Gauss-Bonnet black hole. From the left to the right $\bar{\alpha}=1,5,10$ and 20, respectively, where the light red region corresponds to $\ell=3$ and the light red region plus the light blue region correspond to $\ell=2$.

to destabilize the black hole. Moreover, if $\bar{\alpha}$ is very large, the stability of the black hole is totally determined by the cosmological constant, $L>L_{\ell}$ or $L<L_{\ell}$, but being independent of the charge, which can be seen in figure 6 . In addition, the angular quantum number $\ell$ plays the same role as in the case without the Gauss-Bonnet term or the charge, so $\ell=2$ corresponds to the most unstable mode of the de Sitter charged Gauss-Bonnet black hole.

AdS charged Gauss-Bonnet black hole. The above discussion can be easily applied to the AdS case by replacing $L \rightarrow i L$, except that in this case we require $4 \tilde{\alpha} / L^{2} \leq 1$, or in terms of $\bar{\alpha}$, which is expressed as

$$
\bar{\alpha} \leq \frac{L^{4}}{4\left(1+L^{2}\right)}
$$

in order to have a well-defined vacuum. The analogous effective equations and quasinormal modes can be obtained after such a replacement. From (4.17), after the replacement $L \rightarrow i L$ we obtain the necessary condition for the existence of unstable mode

$$
\begin{aligned}
& L^{4}(\ell-1)\left(1+\bar{\alpha}+2 \bar{\alpha}^{2}+\bar{Q}^{2}(2 \bar{\alpha}-1)\right)+L^{2}\left(1+\bar{Q}^{2}(1-4 \bar{\alpha}+6 \ell \bar{\alpha})+\bar{\alpha}(5-4 \bar{\alpha}+\ell(6 \bar{\alpha}-2) \bar{\alpha})\right. \\
& \left.+2\left(3+\bar{Q}^{2}(2 \ell-1)\right)+2 \ell(\bar{\alpha}-1)-\bar{\alpha}\right) \bar{\alpha}<0 .
\end{aligned}
$$

Then it is evident that the AdS charged Gauss-Bonnet black hole is always stable at large $D$.

Deformed static solution. It was found in [28] that for the de Sitter charged black hole in higher dimensions there exists deformed black hole solution at the edge of the instability. This kind of deformed solution is not spherically symmetric any more. In [25], the deformed solution of the de Sitter charged black hole has been constructed from the large $D$ effective equations. It is interesting to see if there is similar phenomenon for the Gauss-Bonnet gravity.

From the perturbation analysis we notice that the deformed static solution does exist in the Gauss-Bonnet gravity. Since the unstable mode is always of a purely imaginary frequency, at the edge of the instability, there exists a non-trivial zero-mode $(\omega=0)$ static perturbation, suggesting the existence of a non-spherical symmetric static solution branch. By solving the large $D$ effective equations (3.9), (3.10) and (3.11) in the static case, we are 
able to find such a static non-spherical symmetric de Sitter charged Gauss-Bonnet black hole solution. In the background of de Sitter spacetime (4.1), from (3.13) and (3.14) in terms of $\bar{m}(z), \bar{q}(z), p_{z}(z)$ and $\bar{Q}$, the static solution is given by

$$
p_{z}(z)=\bar{m}^{\prime}(z), \quad \bar{q}(z)=\frac{\bar{Q}}{1+\bar{\alpha}+\bar{Q}^{2}} \bar{m}(z), \quad \bar{m}(z)=e^{P(z)},
$$

where

$$
\begin{aligned}
P(z) & =P_{0}+P_{1}(\cos z)^{P_{2}}, \\
P_{2} & =\frac{2 \bar{\alpha}\left(-3+\bar{\alpha}+\bar{Q}^{2}\right)+L^{2}\left(1+\bar{Q}^{2}(1-4 \bar{\alpha})+(5-4 \bar{\alpha}) \bar{\alpha}\right)+L^{4}\left(1+\bar{\alpha}+2 \bar{\alpha}^{2}+\bar{Q}^{2}(-1+2 \bar{\alpha})\right)}{4 \bar{\alpha}\left(-1+\bar{Q}^{2}+\bar{\alpha}\right)-2 L^{2} \bar{\alpha}\left(-1+3 \bar{Q}^{2}+3 \bar{\alpha}\right)+L^{4}\left(1+\bar{\alpha}+2 \bar{\alpha}^{2}+\bar{Q}^{2}(-1+2 \bar{\alpha})\right)} .
\end{aligned}
$$

$P_{0}$ and $P_{1}$ are the integration constants, whose physical meanings become clear after comparing with (4.10). If we set the horizon radius $\mathrm{R}_{+}=1$ then $e^{P_{0}}=1+\bar{\alpha}+\bar{Q}^{2}$, so $P_{0}$ is an $\mathcal{O}(1 / n)$ redefinition of the horizon radius. $P_{1}$ describes an $\mathcal{O}(1 / n)$ amplitude deformed from spherical symmetry. The solutions is not analytic at $z=\pi / 2$ for general $\bar{Q}, \bar{\alpha}$ and $1 / L$. However, at the edge of the instability, the right hand side of (4.17) must be zero such that $P_{2}=\ell$. In this case, the solution becomes regular. For $\ell \geq 2$, the solution is a static solution without spherical symmetry.

\section{The case $\tilde{\alpha}<0$}

Now we discussion the case that the Gauss-Bonnet term has a negative coefficient, i.e. $\tilde{\alpha}<0$. From (3.8) we find a restriction on the value of $\bar{\alpha}$, that is

$$
\bar{\alpha}>-1
$$

otherwise the solution is not well-defined. With this condition it is easy to extend the previous discussion to the case $\bar{\alpha}<0$. Here we focus on the stabilities of the solutions. The necessary condition for the scalar gravitational perturbation to have an unstable mode is given by

$$
\begin{aligned}
& \left(L^{4}+2 L^{2}\left(L^{2}-1\right) \bar{\alpha}\right)\left(2\left(3+\bar{Q}^{2}(2 \ell-1)+2 \ell(\bar{\alpha}-1)-\bar{\alpha}\right) \bar{\alpha}\right. \\
& -L^{2}\left(1+\bar{Q}^{2}(1-4 \bar{\alpha}+6 \ell \bar{\alpha})+\bar{\alpha}(5-4 \bar{\alpha}+\ell(6 \bar{\alpha}-2) \bar{\alpha})\right) \\
& \left.+L^{4}(\ell-1)\left(1+\bar{\alpha}+2 \bar{\alpha}^{2}+\bar{Q}^{2}(2 \bar{\alpha}-1)\right)\right)<0 .
\end{aligned}
$$

Different from the one in (4.17), the first factor in the above expression could be negative, as now $\bar{\alpha}<0$. This makes discussion on the instability of the black hole slightly more complicated than the case $\bar{\alpha}>0$. It is convenient to decompose the above expression into 
two parts

$$
\begin{aligned}
f_{1}(L, \bar{\alpha})= & L^{4}+2 L^{2}\left(L^{2}-1\right) \bar{\alpha} \\
f_{2}(L, \bar{\alpha}, \bar{Q}, \ell)= & \left.2\left(3+\bar{Q}^{2}(2 \ell-1)\right)+2 \ell(\bar{\alpha}-1)-\bar{\alpha}\right) \bar{\alpha} \\
& -L^{2}\left(1+\bar{Q}^{2}(1-4 \bar{\alpha}+6 \ell \bar{\alpha})+\bar{\alpha}(5-4 \bar{\alpha}+\ell(6 \bar{\alpha}-2) \bar{\alpha})\right. \\
& +L^{4}(\ell-1)\left(1+\bar{\alpha}+2 \bar{\alpha}^{2}+\bar{Q}^{2}(2 \bar{\alpha}-1)\right)
\end{aligned}
$$

then the necessary condition (5.2) is equivalent to

$$
f_{1} \cdot f_{2}<0
$$

In other words, the necessary condition for the black hole to develop the unstable mode is either

$$
f_{1}>0, \quad \text { and } \quad f_{2}<0
$$

or

$$
f_{1}<0, \quad \text { and } \quad f_{2}>0 \text {. }
$$

In the former case, the discussion of $f_{2}<0$ is similar to the one for the case $\bar{\alpha}>0$.

Asymptotically flat Gauss-Bonnet black hole. For the asymptotically flat charged Gauss-Bonnet black hole, the necessary condition (5.2) is reduced to

$$
(1+2 \bar{\alpha})\left(1+\bar{\alpha}+2 \bar{\alpha}^{2}+\bar{Q}^{2}(2 \bar{\alpha}-1)\right)<0
$$

from which it is straightforward to read the unstable regions, as shown in figure 7 , where we have taken into account that $\bar{Q}^{2} \leq 1+\bar{\alpha}$. In particular, if $\bar{\alpha}<-1 / 2$, the first factor $1+2 \bar{\alpha}<0$ while the factor in the second bracket is positive definite such that the black hole is always unstable, even when $\bar{Q}=0$. On the other hand, when $\bar{\alpha} \rightarrow 0$, the unstable region becomes more and more smaller. When $\bar{\alpha}=0$ the unstable region becomes vanishing and the black hole becomes stable, which is in accord with the fact that the asymptotical flat charged black hole in the Einstein gravity is always stable.

de Sitter Gauss-Bonnet black hole. For the de Sitter charged Gauss-Bonnet black hole, the region $f_{1}<0$ is given by

$$
-1<\bar{\alpha}<-\frac{L^{2}}{2\left(L^{2}-1\right)} .
$$

Since

$$
-\left.\frac{L^{2}}{2\left(L^{2}-1\right)}\right|_{\max }=-\frac{1}{2},
$$

if $\bar{\alpha} \geq-1 / 2$, then $f_{1} \geq 0$ is always satisfied and the unstable region is determined by $f_{2}<0$. In figure 8 we show the unstable regions of the de Sitter charged Gauss-Bonnet black hole with different values of $\bar{\alpha} \geq-1 / 2$. Similar to the case $\bar{\alpha}>0$, the larger angular quantum number $\ell$ reduces the unstable region as well, and the instability occurs only 


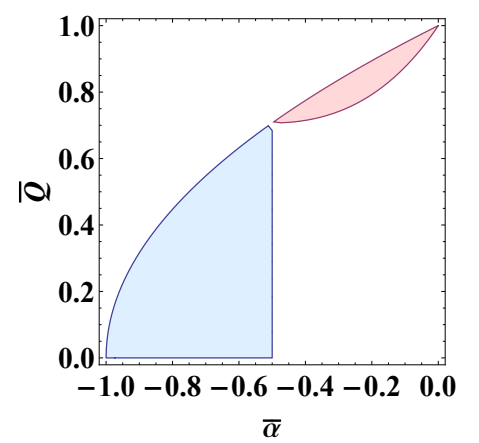

Figure 7. The unstable regions of the asymptotically flat charged Gauss-Bonnet black hole are shown in $(\bar{\alpha}, \bar{Q})$ plane. The light red region corresponds to $f_{2}<0$ and the light blue region corresponds to $f_{1}<0$, since these two regions have no overlap, both of them belong to the unstable regions.
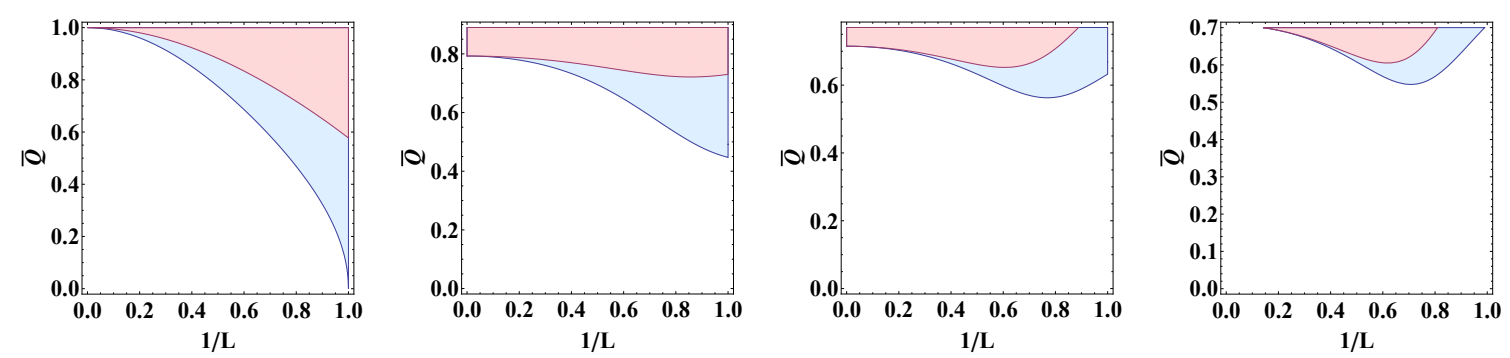

Figure 8. The unstable regions of the de Sitter charged Gauss-Bonnet black hole for $\bar{\alpha} \geq-1 / 2$. From the left to the right $\bar{\alpha}=0,-0.2,-0.4$ and -0.5 , respectively, where the light red region corresponds to $\ell=3$ and the light red region plus the light blue region correspond to $\ell=2$.

when the charge is larger than a critical value. But now as the Gauss-Bonnet coefficient $\bar{\alpha}$ is negative, the range of $\bar{Q}$ shrinks due to the relation (4.9), as one can see from figure 8.

If $\bar{\alpha}<-1 / 2$, then $f_{1}$ could be negative. When $f_{1}$ is negative, as

$$
\left.f_{2}\right|_{\frac{1}{L^{2}}=1+\frac{1}{2 \bar{\alpha}}}=\frac{4(\ell-1)\left(\bar{Q}^{2}-1-\bar{\alpha}\right) \bar{\alpha}}{(1+2 \bar{\alpha})^{2}} \geq 0
$$

so $f_{1}<0$ has no overlap with $f_{2}<0$, both of them belonging to the unstable regions, as shown in figure 9 . For the unstable region $f_{2}<0$, the angular quantum number $\ell$ has the same effect on the instability as the one in the case $\bar{\alpha} \geq-1 / 2$ : the larger $\ell$, the smaller the unstable region. On the other hand, the unstable region $f_{1}<0$ is determined by the cosmological constant $1 / L^{2}$ : the smaller $1 / L^{2}$, the smaller the unstable region.

AdS Gauss-Bonnet black hole. To discuss the instability of the AdS charged GaussBonnet black hole, we just need to replace $L$ by $i L$ as before. The necessary condition for the existence of unstable mode is still given by $f_{1} \cdot f_{2}<0$, with $f_{1}$ and $f_{2}$ being now 


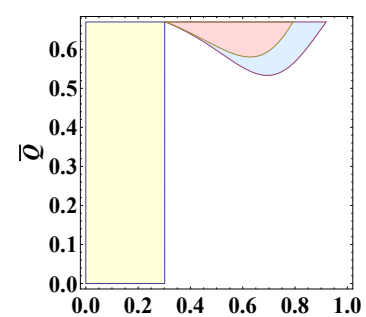

$1 / \mathrm{L}$

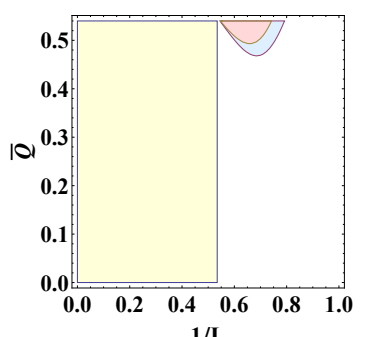

$1 / \mathrm{L}$

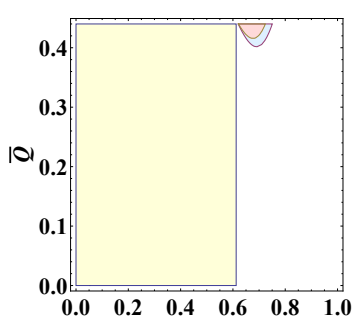

$1 / \mathrm{L}$

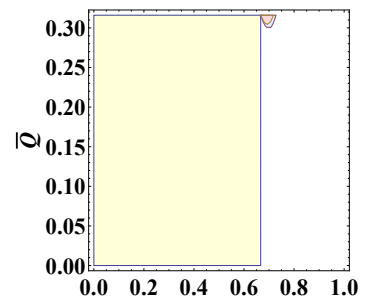

$1 / \mathrm{L}$

Figure 9. The unstable regions of the de Sitter charged Gauss-Bonnet black hole for $\bar{\alpha}<-1 / 2$. From the left to the right $\bar{\alpha}=-0.55,-0.7,-0.8$ and -0.9 , respectively, where the light yellow region corresponds to $f_{1}<0$ and the light red region plus the light blue region correspond to $f_{2}<0$. As in figure 8 , the light red region corresponds to $\ell=3$ and the light red region plus the light blue region correspond to $\ell=2$.

defined by

$$
\begin{aligned}
f_{1}(L, \bar{\alpha})= & L^{4}+2 L^{2}\left(L^{2}+1\right) \bar{\alpha}, \\
f_{2}(L, \bar{\alpha}, \bar{Q}, \ell)= & \left.2\left(3+\bar{Q}^{2}(2 \ell-1)\right)+2 \ell(\bar{\alpha}-1)-\bar{\alpha}\right) \bar{\alpha}, \\
& +L^{2}\left(1+\bar{Q}^{2}(1-4 \bar{\alpha}+6 \ell \bar{\alpha})+\bar{\alpha}(5-4 \bar{\alpha}+\ell(6 \bar{\alpha}-2) \bar{\alpha})\right. \\
& +L^{4}(\ell-1)\left(1+\bar{\alpha}+2 \bar{\alpha}^{2}+\bar{Q}^{2}(2 \bar{\alpha}-1)\right) .
\end{aligned}
$$

The region $f_{1}<0$ is given by

$$
-1<\bar{\alpha}<-\frac{L^{2}}{2\left(L^{2}+1\right)}
$$

as now we only demand $L^{2}>0$, then

$$
-\left.\frac{L^{2}}{2\left(L^{2}+1\right)}\right|_{\min }=-\frac{1}{2}
$$

If $\bar{\alpha} \leq-1 / 2$, then $f_{1} \leq 0$ is always satisfied, and in this case the unstable region is completely determined by $f_{2}>0$. It turns out that as long as $\bar{\alpha} \leq-1 / 2, f_{2}>0$ is always satisfied for the whole range of the parameters. This can be seen as follows, from (5.13) we observe that the coefficients of the linear $\bar{Q}^{2}$ terms are all negative, so

$$
\begin{aligned}
f_{2} & \geq f_{2}\left(Q^{2}=1+\bar{\alpha}\right) \\
& =2\left(L^{2}+2\left(1+L^{2}\right) \bar{\alpha}\right)\left(1+\left(-1+L^{2}(\ell-1)+2 \ell\right) \bar{\alpha}\right) .
\end{aligned}
$$

It is easy to determine that the minimum of the right hand side of the above expression occurs at $\bar{\alpha}=-1 / 2$, such that

$$
f_{2} \geq f_{2}\left(Q^{2}=1+\bar{\alpha}, \bar{\alpha}=-1 / 2\right)=L^{2}(\ell-1)+2 \ell-3>0 .
$$

If $\bar{\alpha} \geq-1 / 2$, different from the discussion in the de Sitter case, $f_{1}<0$ and $f_{2}<0$ have overlapping region when both $L$ and $|\alpha|$ are small, as shown in figure 10. Such overlapping 

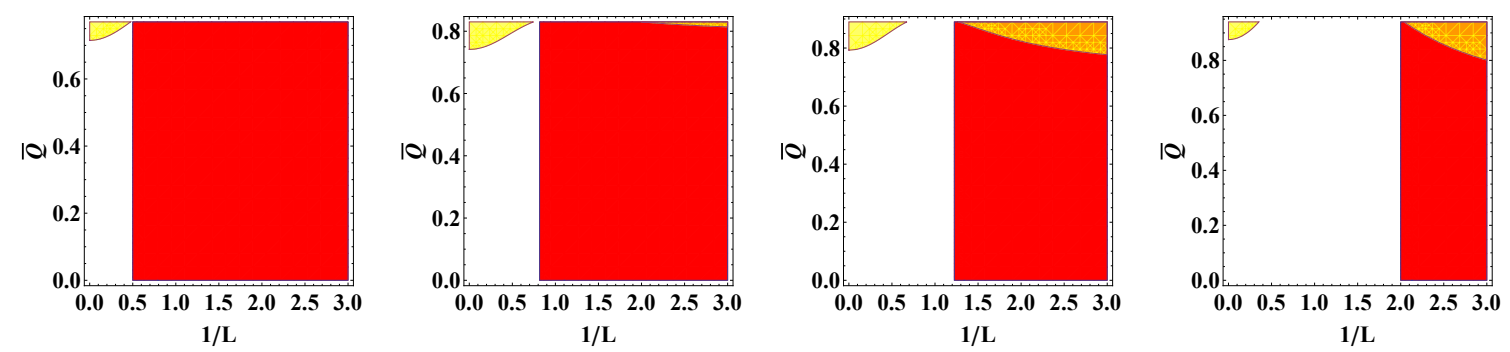

Figure 10. The unstable regions of the AdS charged Gauss-Bonnet black hole. From the left to the right $\bar{\alpha}=-0.4,-0.3,-0.2$ and -0.1 , respectively, where the red region corresponds to $f_{1}<0$, the yellow region correspond to $f_{2}<0$ and the orange region is the overlap which belongs to the stable region. Here we take $\ell=2$.
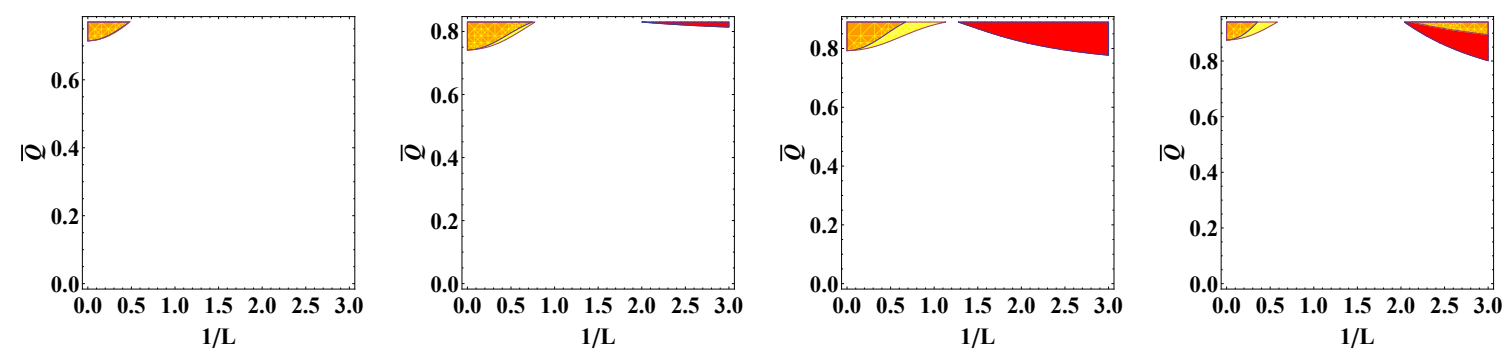

Figure 11. The region $f_{2}<0$ with $\ell=2$ and $\ell=3$. From the left to the right $\bar{\alpha}=-0.4,-0.3,-0.2$ and -0.1 , respectively, where the red region corresponds to $\ell=2$, the yellow region correspond to $\ell=3$. The upper left corner shows that a larger $\ell$ leads to a larger unstable region. On the contrary the right upper corner shows that a larger $\ell$ corresponds to a smaller region. However, the right upper corner is the region overlapping with $f_{1}<0$ so that it actually belongs to the stable region. In any case, a larger $\ell$ always leads to a smaller unstable region.

region corresponds to stable black holes. To see the effect of the angular quantum number $\ell$ on the instability, we depict the region $f_{2}<0$ with different $\ell$ in figure 11 , from which we can see that a larger $\ell$ leads to a larger unstable region. This is different from the case with a positive Gauss-Bonnet term, in which $\ell=2$ has the largest unstable region.

It would be interesting to consider the $\bar{\alpha} \rightarrow 0^{-}$limit. According the previous discussion, when $\bar{\alpha} \geq 0$, the AdS Reissner-Nordstrom black holes are always stable. Actually, from figure 10 we can see that as $\bar{\alpha}$ turns to zero, the unstable regions shrinks. This can be seen more clearly in figure 12 , where we depict the unstable regions with $\bar{\alpha}$ being close to zero. From figure 12 we can see that when the range of $L$ is fixed, as $\bar{\alpha} \rightarrow 0$ the unstable region shrinks to zero. However, for a fixed tiny $\bar{\alpha}$ as long as $L$ is sufficient small the unstable region always exists. But we note that the validity of the $1 / n$ expansion requires that $1 / L$ to be finite, so $L$ cannot be arbitrary small. Therefore the $\bar{\alpha} \rightarrow 0$ limit is in accord with the fact that the AdS Reissner-Nordstrom black holes are always stable.

Deformed static solution. The discussion of the deformed static solution in the previous section can be naturally extended to the case with a negative Gauss-Bonnet term. Since in this case the unstable mode is also of a pure imaginary frequency, so identically at the edge of the instability there exists a non-trivial zero-mode static perturbation indi- 

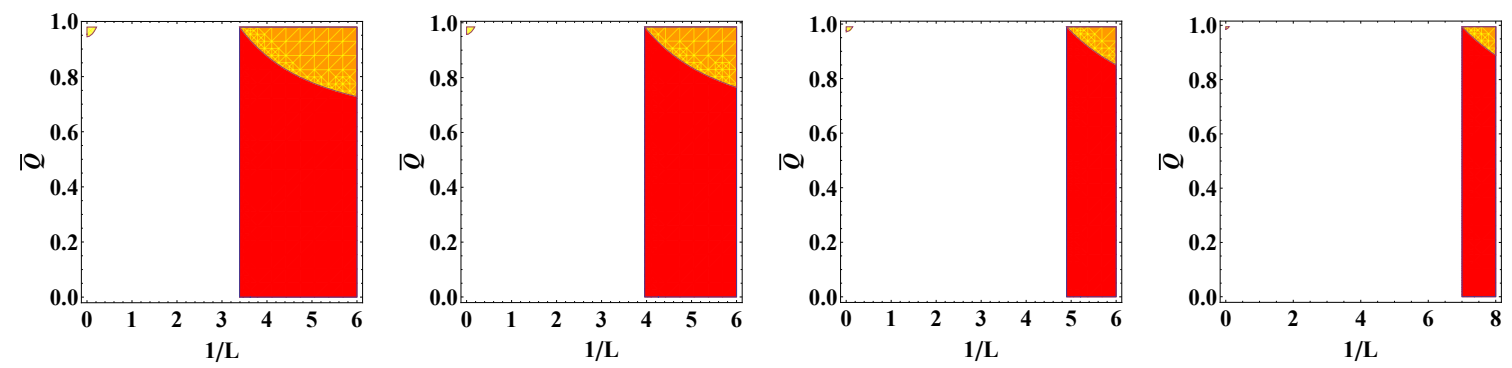

Figure 12. The unstable regions of the AdS charged Gauss-Bonnet black hole. From the left to the right $\bar{\alpha}=-0.04,-0.03,-0.02$ and -0.01 , respectively, where the red region corresponds to $f_{1}<0$, the yellow region correspond to $f_{2}<0$ and the orange region is their overlap which belongs to the stable region. Here we take $\ell=2$.

cating the existence of a non-spherical symmetrical symmetric static solution branch. By solving the large $D$ effective equations in the static case, we can find such a static solution as well. The solution is also given by (4.31) and (4.32), where $P_{2}$ is given by (4.33) when the black hole is in the de Sitter spacetime, and $P_{2}=1$ or

$$
P_{2}=\frac{2 \bar{\alpha}\left(-3+\bar{\alpha}+\bar{Q}^{2}\right)-L^{2}\left(1+\bar{Q}^{2}(1-4 \bar{\alpha})+(5-4 \bar{\alpha}) \bar{\alpha}\right)+L^{4}\left(1+\bar{\alpha}+2 \bar{\alpha}^{2}+\bar{Q}^{2}(-1+2 \bar{\alpha})\right)}{4 \bar{\alpha}\left(-1+\bar{Q}^{2}+\bar{\alpha}\right)+2 L^{2} \bar{\alpha}\left(-1+3 \bar{Q}^{2}+3 \bar{\alpha}\right)+L^{4}\left(1+\bar{\alpha}+2 \bar{\alpha}^{2}+\bar{Q}^{2}(-1+2 \bar{\alpha})\right)},
$$

when the background spacetime is asymptotically flat or AdS correspondingly.

\section{Summary}

In this article we studied static Gauss-Bonnet black holes at large $D$. For generality, we considered the black holes in the Einstein-Maxwell-Gauss-Bonnet theory with a cosmological constant. The black holes include the charged black hole in the asymptotically flat and (A)dS spacetimes. After deriving the large $D$ effective equations of the black hole to the next-leading order of $1 / D$, we showed that the static black hole could still be the solution of an elastic theory. Actually we found the relation $\sqrt{-g_{v v}} \mathcal{K}=$ constant for the membrane embedding. Different from the Einstein gravity, the constant in the relation is not simply the surface gravity. Moreover, by considering the embedding of the membrane in the spherical coordinates, we read the static solutions and furthermore investigated their instabilities.

Considering the perturbation around the exact solutions, we could read the charge and scalar-type quasinormal modes of the static Gauss-Bonnet black holes analytically. The results we got can be summarized as following. If the Gauss-Bonnet term is positive, the black holes could be stable or unstable, depending on the cosmological constant, charge and the Gauss-Bonnet coefficient.

1. For the static asymptotically flat Gauss-Bonnet black hole, it is always stable, no matter it is charged or not, and no matter how large the Gauss-Bonnet parameter is. This answers the unsolved question on the stability of the Gauss-Bonnet black hole 
studied in [20] in which only the black hole with a small or a large Gauss-Bonnet parameter has shown to be stable.

2. For the static asymptotically AdS Gauss-Bonnet black hole, it is always stable, no matter it is charged or not.

3. For the static asymptotically de Sitter Gauss-Bonnet black hole, it becomes unstable when the cosmological constant is sufficiently large. This kind of $\Lambda$-instability is very similar to the one of de Sitter Reissner-Nordstrom black hole. And similar to the de Sitter Reissner-Nordstrom black hole, the charge may enhance the instability. However, the presence of the Gauss-Bonnet term does make difference. When the cosmological constant is small, the term helps to stabilize the black hole such that the black hole could be stable no matter how large the charge is. When the cosmological constant is large, on the contrary the term helps to destabilize the black hole such that the unstable region is enlarged. When the Gauss-Bonnet coefficient is very large, the stability of the black hole is determined by a critical cosmological constant which is independent of the charge.

4. At the marginal lines of instabilities there exists a non-trivial zero-mode static perturbation. This suggests the existence of a non-spherically symmetric static charged de Sitter Gauss-Bonnet black hole solution branch, which can be constructed by solving the large $D$ effective equations.

On the other hand, if the Gauss-Bonnet term is negative, the stability of the black hole is slightly more complex. The presence of such a negative term would make the black holes unstable. It turns out that the Gauss-Bonnet black hole could be unstable no matter the spacetime is asymptotically flat or (A)dS. Especially, if $\bar{\alpha}<-1 / 2$, the asymptotically flat and AdS Gauss-Bonnet black holes are always unstable for the whole range of the parameters. But as the parameter $\bar{\alpha}$ turns to zero, the unstable regions shrink to zero for the asymptotically flat and AdS black holes. Furthermore, similar to the case with a positive Gauss-Bonnet term, there exists a non-spherically symmetric static black hole solution branch as well.

The work in this paper can be extended in several directions. For example, it is possible to study Gauss-Bonnet black branes by using the large $D$ expansion method. Up to now the Gauss-Bonnet black brane has not been known in a closed form. The large $D$ expansion method may provide a potential tool to construct the analytical solution and help to study the phase structure and the Gregory-Laflamme instability [29, 30]. The similar discussions have already been made in the Einstein gravity [10, 12-14, 31]. It might also be interesting to extend the study to the rotating cases, just like the ones in the Einstein gravity [7, 32, 33]. In the Gauss-Bonnet gravity, the construction of the rotating black holes is still an open question. We wish the large $D$ analysis may shed light on this interesting issue. Moreover, it is certainly interesting to address various issues of the Gauss-Bonnet black holes in the framework of membrane paradigm developed in [34-38]. 


\section{Acknowledgments}

The work was in part supported by NSFC Grant No. 11275010, No. 11335012 and No. 11325522.

Open Access. This article is distributed under the terms of the Creative Commons Attribution License (CC-BY 4.0), which permits any use, distribution and reproduction in any medium, provided the original author(s) and source are credited.

\section{References}

[1] R. Emparan, R. Suzuki and K. Tanabe, The large D limit of General Relativity, JHEP 06 (2013) 009 [arXiv: 1302.6382] [INSPIRE].

[2] R. Emparan, D. Grumiller and K. Tanabe, Large-D gravity and low-D strings, Phys. Rev. Lett. 110 (2013) 251102 [arXiv: 1303.1995] [INSPIRE].

[3] R. Emparan and K. Tanabe, Universal quasinormal modes of large D black holes, Phys. Rev. D 89 (2014) 064028 [arXiv: 1401.1957] [InSPIRE].

[4] R. Emparan, R. Suzuki and K. Tanabe, Quasinormal modes of (Anti-)de Sitter black holes in the 1/D expansion, JHEP 04 (2015) 085 [arXiv: 1502.02820] [INSPIRE].

[5] R. Emparan, R. Suzuki and K. Tanabe, Decoupling and non-decoupling dynamics of large D black holes, JHEP 07 (2014) 113 [arXiv:1406.1258] [INSPIRE].

[6] R. Emparan, T. Shiromizu, R. Suzuki, K. Tanabe and T. Tanaka, Effective theory of Black Holes in the 1/D expansion, JHEP 06 (2015) 159 [arXiv:1504.06489] [INSPIRE].

[7] R. Suzuki and K. Tanabe, Stationary black holes: Large D analysis, JHEP 09 (2015) 193 [arXiv: 1505.01282] [INSPIRE].

[8] S. Bhattacharyya, A. De, S. Minwalla, R. Mohan and A. Saha, A membrane paradigm at large D, JHEP 04 (2016) 076 [arXiv: 1504.06613] [INSPIRE].

[9] S. Bhattacharyya, M. Mandlik, S. Minwalla and S. Thakur, A Charged Membrane Paradigm at Large D, JHEP 04 (2016) 128 [arXiv:1511.03432] [INSPIRE].

[10] R. Emparan, R. Suzuki and K. Tanabe, Evolution and End Point of the Black String Instability: Large D Solution, Phys. Rev. Lett. 115 (2015) 091102 [arXiv:1506.06772] [INSPIRE].

[11] K. Tanabe, Black rings at large D, JHEP 02 (2016) 151 [arXiv:1510.02200] [INSPIRE].

[12] R. Suzuki and K. Tanabe, Non-uniform black strings and the critical dimension in the $1 / D$ expansion, JHEP 10 (2015) 107 [arXiv:1506. 01890] [INSPIRE].

[13] R. Emparan, K. Izumi, R. Luna, R. Suzuki and K. Tanabe, Hydro-elastic Complementarity in Black Branes at large D, JHEP 06 (2016) 117 [arXiv:1602.05752] [INSPIRE].

[14] M. Rozali and A. Vincart-Emard, On Brane Instabilities in the Large D Limit, JHEP 08 (2016) 166 [arXiv: 1607.01747] [INSPIRE].

[15] B. Zwiebach, Curvature Squared Terms and String Theories, Phys. Lett. B 156 (1985) 315 [INSPIRE].

[16] D. G. Boulware and S. Deser, String-Generated Gravity Models, Phys. Rev. Lett. 55 (1985) 2656 [INSPIRE]. 
[17] J.T. Wheeler, Symmetric Solutions to the Gauss-Bonnet Extended Einstein Equations, Nucl. Phys. B 268 (1986) 737 [INSPIRE].

[18] D.L. Wiltshire, Spherically symmetric solutions of Einstein-Maxwell theory with a Gauss-Bonnet term, Phys. Lett. 169B (1986) 36 [InSPIRE].

[19] D.L. Wiltshire, Black holes in string generated gravity models, Phys. Rev. D 38 (1988) 2445 [INSPIRE].

[20] B. Chen, Z.-Y. Fan, P. Li and W. Ye, Quasinormal modes of Gauss-Bonnet black holes at large D, JHEP 01 (2016) 085 [arXiv: 1511.08706] [INSPIRE].

[21] G. Dotti and R.J. Gleiser, Linear stability of Einstein-Gauss-Bonnet static spacetimes. Part I. Tensor perturbations, Phys. Rev. D 72 (2005) 044018 [gr-qc/0503117] [InSPIRE].

[22] R.J. Gleiser and G. Dotti, Linear stability of Einstein-Gauss-Bonnet static spacetimes. Part II: Vector and scalar perturbations, Phys. Rev. D 72 (2005) 124002 [gr-qc/0510069] [INSPIRE].

[23] R.A. Konoplya and A. Zhidenko, (In)stability of D-dimensional black holes in Gauss-Bonnet theory, Phys. Rev. D 77 (2008) 104004 [arXiv:0802.0267] [inSPIRE].

[24] M.A. Cuyubamba, R.A. Konoplya and A. Zhidenko, Quasinormal modes and a new instability of Einstein-Gauss-Bonnet black holes in the de Sitter world, Phys. Rev. D 93 (2016) 104053 [arXiv: 1604.03604] [INSPIRE].

[25] K. Tanabe, Instability of the de Sitter Reissner-Nordstrom black hole in the $1 / D$ expansion, Class. Quant. Grav. 33 (2016) 125016 [arXiv:1511.06059] [INSPIRE].

[26] R.C. Myers and M.J. Perry, Black Holes in Higher Dimensional Space-Times, Annals Phys. 172 (1986) 304 [INSPIRE].

[27] B. Chen, P.-C. Li and Z.-z. Wang, Charged Black Rings at large D, arXiv:1702.00886 [INSPIRE].

[28] R.A. Konoplya and A. Zhidenko, Instability of higher dimensional charged black holes in the de-Sitter world, Phys. Rev. Lett. 103 (2009) 161101 [arXiv:0809.2822] [INSPIRE].

[29] R. Gregory and R. Laflamme, Black strings and p-branes are unstable, Phys. Rev. Lett. 70 (1993) 2837 [hep-th/9301052] [INSPIRE].

[30] R. Gregory and R. Laflamme, The Instability of charged black strings and p-branes, Nucl. Phys. B 428 (1994) 399 [hep-th/9404071] [INSPIRE].

[31] A. Sadhu and V. Suneeta, Nonspherically symmetric black string perturbations in the large dimension limit, Phys. Rev. D 93 (2016) 124002 [arXiv:1604.00595] [INSPIRE].

[32] R. Emparan, R. Suzuki and K. Tanabe, Instability of rotating black holes: large D analysis, JHEP 06 (2014) 106 [arXiv: 1402.6215] [INSPIRE].

[33] K. Tanabe, Charged rotating black holes at large D, arXiv:1605.08854 [INSPIRE].

[34] S. Bhattacharyya, A. De, S. Minwalla, R. Mohan and A. Saha, A membrane paradigm at large D, JHEP 04 (2016) 076 [arXiv: 1504.06613] [INSPIRE].

[35] S. Bhattacharyya, M. Mandlik, S. Minwalla and S. Thakur, A Charged Membrane Paradigm at Large D, JHEP 04 (2016) 128 [arXiv: 1511.03432] [INSPIRE]. 
[36] Y. Dandekar, A. De, S. Mazumdar, S. Minwalla and A. Saha, The large D black hole Membrane Paradigm at first subleading order, JHEP 12 (2016) 113 [arXiv:1607.06475] [INSPIRE].

[37] Y. Dandekar, S. Mazumdar, S. Minwalla and A. Saha, Unstable 'black branes' from scaled membranes at large D, JHEP 12 (2016) 140 [arXiv:1609.02912] [INSPIRE].

[38] S. Bhattacharyya, A.K. Mandal, M. Mandlik, U. Mehta, S. Minwalla, U. Sharma et al., Currents and Radiation from the large D Black Hole Membrane, arXiv:1611.09310 [INSPIRE].

[39] R.A. Konoplya and A. Zhidenko, Eikonal instability of Gauss-Bonnet-(anti-)de Sitter black holes, arXiv: 1701.01652 [INSPIRE].

[40] P.A. González, R.A. Konoplya and Y. Vásquez, Quasinormal modes of a scalar field in the Einstein-Gauss-Bonnet-AdS black hole background: perturbative and non-perturbative branches, arXiv:1703.06215 [INSPIRE]. 